International Journal of Pure and Applied Mathematics

Volume 93 No. 6 2014, 879-883

ISSN: 1311-8080 (printed version); ISSN: 1314-3395 (on-line version)

url: http://www.ijpam.eu

doi: http://dx.doi.org/10.12732/ijpam.v93i6.11

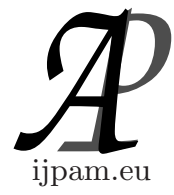

\title{
GENERAL SOLUTION TO BATEMAN'S DIFFERENTIAL EQUATIONS WITH DIRECT INDEX NOTATION
}

\author{
Ronaldo Thibes $^{1 \S}$, Suzicleide L. de Oliveira ${ }^{2}$ \\ 1,2 Departamento de Ciências Exatas e Naturais \\ Universidade Estadual do Sudoeste da Bahia \\ Itapetinga - BA, BRAZIL
}

\begin{abstract}
Bateman's equations describe a system of radioactive decay of different products in chain. We provide an explicit proof of the general solution to Bateman's equations by direct attack. By using index notation of ordinary sums and products the system of $k$ differential equations can be shown to be easily solved. We compare our method with previous more elaborated ones published in the literature.
\end{abstract}

AMS Subject Classification: 34A05, 81V35

Key Words: differential equations, ode, nuclear decay, index notation

\section{Motivation}

In a well known ancient paper, Bateman solved a system of real coupled linear first order ordinary differential equations describing the radioactive decay and transmutation among different atomic nuclei [1]. In that paper Bateman used Laplace transformations and complex integrals to achieve the solution. A modern version of Bateman's approach can be found in [2]. Nowadays everyone knows that the solution to a system of linear first order differential equations in principle can be obtained immediately by diagonalizing and inverting a square matrix as can be seen in any textbook on differential equations. In fact, in

Received: April 23, 2014

(c) 2014 Academic Publications, Ltd.

$\S$ Correspondence author url: www.acadpubl.eu 
2002, Moral and Pacheco published the standard matrix solution of this problem in the American Journal of Physics [3]. However, we feel the problem is simple enough to be attacked directly in a recursive way. We show that by using common index notations of sum and product a natural general solution can be conjectured and proved to be correct.

\section{The Problem and a Particular Case}

Bateman's system of differential equations can be written as

$$
\frac{d F_{i}}{d x}(x)=\lambda_{i-1} F_{i-1}(x)-\lambda_{i} F_{i}(x),
$$

with $i=1, \ldots, k, \lambda_{0}=\lambda_{k}=0$ and $k \geq 2$. The $F_{i}$ 's are real differentiable functions of the real variable $x$ while the parameters $\lambda_{i}$, assumed to be strictly distinct among themselves, represent the decay rates. Physically $F_{i}$ represents the amount of substance $i$ which decays into substance $i+1$ with decay rate $\lambda_{i}$, for $i<k$. The last substance $F_{k}$ is considered stable, thus $\lambda_{k} \equiv 0$ and

$$
\frac{d}{d x} \sum_{i=1}^{k} F_{i}(x)=0
$$

ensures the conservation of the total amount of substance.

In order to get a feeling for the problem let us consider the case $k=4$. The index $i$ running from 1 to 4 , the first equation of (1) leads to the trivial solution

$$
F_{1}(x)=A_{1} e^{-\lambda_{1} x},
$$

with $A_{1}$ an integration constant. Now we substitute $F_{1}$ into the second equation of (1), solve for $F_{2}(x)$ proceeding this way so on and so forth up to the fourth equation, obtaining

$$
\begin{aligned}
& F_{2}(x)=\frac{\lambda_{1}}{\lambda_{2}-\lambda_{1}} A_{1} e^{-\lambda_{1} x}+A_{2} e^{-\lambda_{2} x} \\
& F_{3}(x)=\frac{\lambda_{2} \lambda_{1}}{\left(\lambda_{2}-\lambda_{1}\right)\left(\lambda_{3}-\lambda_{1}\right)} A_{1} e^{-\lambda_{1} x}+\frac{\lambda_{2}}{\lambda_{3}-\lambda_{2}} A_{2} e^{-\lambda_{2} x}+A_{3} e^{-\lambda_{3} x}, \\
& F_{4}(x)=-\frac{\lambda_{2} \lambda_{3} A_{1} e^{-\lambda_{1} x}}{\left(\lambda_{2}-\lambda_{1}\right)\left(\lambda_{3}-\lambda_{1}\right)}-\frac{\lambda_{3} A_{2} e^{-\lambda_{2} x}}{\lambda_{3}-\lambda_{2}}-A_{3} e^{-\lambda_{3} x}+A_{4} .
\end{aligned}
$$

A clear regularity immediately stands out and we may say the general problem is manifestly solved as the proposition of the next section shows. 


\section{The General Solution}

In this section we present and prove the solution to Bateman's equations.

Proposition. The solution to the system of differential equations (1) reads

$$
\begin{aligned}
& F_{i}(x)=\sum_{j=1}^{i} A_{j} e^{-\lambda_{\mathrm{j}} x} \prod_{l=j}^{l=i-1} \frac{\lambda_{l}}{\lambda_{l+1}-\lambda_{j}}, \quad i<k, \\
& F_{k}(x)=\sum_{j=1}^{k} A_{j} e^{-\lambda_{\mathrm{j}} x} \prod_{l=j}^{l=k-2} \frac{-\lambda_{l+1}}{\lambda_{l+1}-\lambda_{j}},
\end{aligned}
$$

where $A_{j}$, with $j=1, \ldots, k$, denote $k$ arbitrary integration constants.

Proof. Elementary substitution of (5) into (1) does the job. In fact for $i<k$ we have for the righthand side of (1)

$$
\begin{aligned}
\text { RHS } \equiv & \lambda_{i-1} F_{i-1}-\lambda_{i} F_{i}, \\
= & \sum_{j=1}^{i-1} A_{j} e^{-\lambda_{\mathrm{j}} x}\left[\lambda_{i-1}\left(\prod_{l=j}^{l=i-2} \frac{\lambda_{l}}{\lambda_{l+1}-\lambda_{j}}\right)-\lambda_{i} \prod_{l=j}^{l=i-1} \frac{\lambda_{l}}{\lambda_{l+1}-\lambda_{j}}\right] \\
& -\lambda_{i} A_{i} e^{-\lambda_{\mathrm{i}} x}
\end{aligned}
$$

where the last term of the sum in $\lambda_{i} F_{i}$ has been explicitly spilled out from the summation sign which now runs up to $i-1$. Now multiply numerator and denominator of the first term within the brackets by $\left(\lambda_{i}-\lambda_{j}\right)$ to write

$$
\begin{aligned}
R H S= & \sum_{j-1}^{i-1} A_{j} e^{-\lambda_{\mathrm{j}} x}\left[\frac{\lambda_{i} \lambda_{i-1}}{\lambda_{i}-\lambda_{j}} \prod_{l=j}^{l=i-2} \frac{\lambda_{l}}{\lambda_{l+1}-\lambda_{j}}+\right. \\
& \left.-\frac{\lambda_{j} \lambda_{i-1}}{\lambda_{i}-\lambda_{j}} \prod_{l=j}^{l=i-2} \frac{\lambda_{l}}{\lambda_{l+1}-\lambda_{j}}-\lambda_{i} \prod_{l=j}^{l=i-1} \frac{\lambda_{l}}{\lambda_{l+1}-\lambda_{j}}\right] \\
& -\lambda_{i} A_{i} e^{-\lambda_{i} x} .
\end{aligned}
$$

Note that the first and last term within the brackets cancel each other while the factor $\lambda_{i-1} /\left(\lambda_{i}-\lambda_{j}\right)$ maybe included in the middle product by letting the index $l$ run from $j$ to $i-1$ leading to

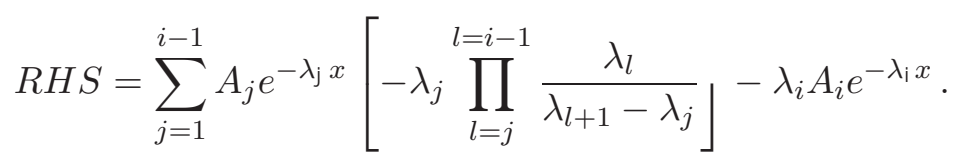


Finally the last term may be included in the summation as

$$
R H S=\sum_{j=1}^{i}\left(-\lambda_{j} A_{j} e^{-\lambda_{\mathrm{j}} x}\right) \prod_{l=j}^{l=i-1} \frac{\lambda_{l}}{\lambda_{l+1}-\lambda_{j}} .
$$

On the other hand, the derivative on the lefthand side of (1) trivially pushes a $-\lambda_{j}$ down for each term in the summation and can be written as

$$
\frac{d F_{i}}{d x}=\sum_{j=1}^{i}\left(-\lambda_{j} A_{j} e^{-\lambda_{\mathrm{j}} x}\right) \prod_{l=j}^{l=i-1} \frac{\lambda_{l}}{\lambda_{l+1}-\lambda_{j}}
$$

confirming (1) as a identity for $i<k$. It remains to verify the last equation of (1), namely,

$$
\frac{d F_{k}(x)}{d x}=\lambda_{k-1} F_{k-1}(x) .
$$

The derivative of $F_{k}$ given in (5) reads explicitly

$$
\frac{d F_{k}(x)}{d x}=\sum_{j=1}^{k} \lambda_{j} A_{j} e^{-\lambda_{\mathrm{j}} x} \prod_{l=j}^{l=k-2} \frac{\lambda_{l+1}}{\lambda_{l+1}-\lambda_{j}} .
$$

Since $\lambda_{k}=0$, the last term in the summation above is null, and the sum may be carried out only up to $j=k-1$. Using elementary properties of products of real numbers we may then rewrite

$$
\begin{aligned}
\frac{d F_{k}(x)}{d x} & =\sum_{j=1}^{k-1} A_{j} e^{-\lambda_{\mathrm{j}} x} \frac{\lambda_{j}\left(\prod_{l=j}^{l=k-2} \lambda_{l+1}\right)}{\prod_{l=j}^{l=k-2}\left(\lambda_{l+1}-\lambda_{j}\right)}, \\
& =\sum_{j=1}^{k-1} A_{j} e^{-\lambda_{\mathrm{j}} x} \frac{\lambda_{k-1} \prod_{l=j-1}^{l=k-3} \lambda_{l+1}}{\prod_{l=j}^{l=k-2}\left(\lambda_{l+1}-\lambda_{j}\right)} .
\end{aligned}
$$

Now redefining the index $l \rightarrow l-1$ in the product of the numerator we get

$$
\begin{aligned}
\frac{d F_{k}(x)}{d x} & =\sum_{j=1}^{k-1} A_{j} e^{-\lambda_{\mathrm{j}} x} \frac{\lambda_{k-1} \prod_{l=j}^{l=k-2} \lambda_{l}}{\prod_{l=j}^{l=k-2}\left(\lambda_{l+1}-\lambda_{j}\right)} \\
& =\lambda_{k} \sum_{j=1}^{k-1} A_{j} e^{-\lambda_{\mathrm{j}} x} \prod_{l=j}^{l=k-2} \frac{\lambda_{l}}{\lambda_{l+1}-\lambda_{j}} \\
& =\lambda_{k-1} F_{k-1}
\end{aligned}
$$

which closes the proof. 
We see thus that this proposition solves completely problem (1), being proved in a thoroughly direct form.

\section{Conclusion}

We have constructed by direct inspection an expression for the functions $F_{i}(x)$ which we proved by explicit and simple substitution to be the general solution to Bateman's equations. We stress that, contrary to [1] and [2] we made no use of Laplace transformations or complex integrals. Although the general matrix method for first order linear systems could be successfully used here as shown by Moral and Pacheco in [3], in the present peculiar case the solution splits out with such a manifest regularity that the presented method of direct attack with index notation for sums and products renders itself simpler.

\section{References}

[1] H. Bateman, Solution of a system of differential equations occurring in the theory of radiactive transformations, Proc. Cambridge Philos. Soc., 15 (1910), 423-427.

[2] D.S. Pressyanov, Short solution of the radioactive chain equations, Am. J. Phys., 70 (2002), 444-445.

[3] L. Moral, A. F. Pacheco, Algebraic approach to the radioactive decay equations, Am. J. Phys., 71 (2003), 684-686. 
